\title{
THE ENVELOPE OF A SUBCATEGORY IN TOPOLOGY AND GROUP THEORY
}

\author{
AHMED AYACHE AND OTHMAN ECHI
}

Received 31 October 2004 and in revised form 5 October 2005

A collection of results are presented which are loosely centered around the notion of reflective subcategory. For example, it is shown that reflective subcategories are orthogonality classes, that the morphisms orthogonal to a reflective subcategory are precisely the morphisms inverted under the reflector, and that each subcategory has a largest "envelope" in the ambient category in which it is reflective. Moreover, known results concerning the envelopes of the category of sober spaces, spectral spaces, and jacspectral spaces, respectively, are summarized and reproved. Finally, attention is focused on the envelopes of one-object subcategories, and examples are considered in the category of groups.

\section{Introduction}

Reflective subcategories arise throughout mathematics, via several examples such as the free group, free ring, functors in algebra, various compactification functors in topology, and completion functors in analysis, and so forth (cf. [25, page 90]). Recall from [25, page 89] that a subcategory $\mathbf{D}$ of a category $\mathbf{C}$ is called reflective (in $\mathbf{C}$ ) if the inclusion functor $\mathbf{I}: \mathbf{D} \rightarrow \mathbf{C}$ has a left adjoint functor $\mathbf{F}: \mathbf{C} \rightarrow \mathbf{D}$; that is, if for each object $A$ of $\mathbf{C}$, there exist an object $\mathbf{F}(A)$ of $\mathbf{D}$ and a morphism $\mu_{A}: A \rightarrow \mathbf{F}(A)$ in $\mathbf{C}$ such that for each object $X$ in $\mathbf{D}$ and each morphism $f: A \rightarrow X$ in $\mathbf{C}$, there exists a unique morphism $\tilde{f}: F(A) \rightarrow X$ in $\mathbf{D}$ such that $\tilde{f} \circ \mu_{A}=f$.

Note that all subcategories considered in this paper are assumed to be full and isomorphism-closed.

The concept of reflections in categories has been investigated by several authors (see, e.g., $[4,5,6,7,8,9,13,14,17,18,19,20,24,29,30,31])$. This concept serves the purpose of unifying various constructions in mathematics.

Historically, the concept of reflections in categories seems to have its origin in the universal extension property of the Stone-Čech compactification of a completely regular $T_{2}$-space.

Notice that all nonexplained notions are standard as in [25]. 
A morphism $f: A \rightarrow B$ and an object $X$ in a category $C$ are called orthogonal [15] if the mapping $\operatorname{hom}_{\mathbf{C}}(f, X): \operatorname{hom}_{\mathbf{C}}(B, X) \rightarrow \operatorname{hom}_{\mathbf{C}}(A, X)$ which takes $g$ to $g f$ is bijective. For a class of morphisms $\Sigma$ (resp., a class of objects D), we denote by $\Sigma^{\perp}$ the class of objects orthogonal to every $f$ in $\Sigma$ (resp., denote by $\mathbf{D}^{\perp}$ the class of morphisms orthogonal to all $X$ in D) [15].

Over the years, reflective subcategories have been studied extensively. It is worth noting that in a remarkable paper [6], Cassidy et al. have given an important study of reflective subcategories.

The present paper is a contribution to the study of reflective subcategories.

We state the following three natural questions. Let $\mathbf{D}$ be a reflective subcategory in $\mathbf{C}$.

Question 1.1. For two objects $A, B$ of $\mathbf{C}$, characterize the set $\operatorname{hom}(A, B) \cap \mathbf{D}^{\perp}$.

The following question is credited to [15].

Question 1.2. Is $\mathbf{D}^{\perp \perp}=\mathbf{D}$ ?

Let $\mathbf{C}$ be a category and $\mathbf{D}$ a subcategory of $\mathbf{C}$ closed under isomorphisms. It is easily seen that there is a largest full subcategory of $\mathbf{C}$ (the envelope of $\mathbf{D}$ ) in which $\mathbf{D}$ is a reflective subcategory.

Question 1.3. How envelopes of various categories can be described in concrete cases?

Note that in [19], Herrlich has considered the envelope question in some generality.

Question 1.1 has been answered by Casacuberta et al. in [5].

The second section of this paper is devoted to collect some information about Questions 1.1 and 1.2 .

The third section deals with an example of orthogonal class of morphisms.

The fourth, fifth, and sixth sections deal with Question 1.3. Note that this question has been tackled long ago (in the setting of HAUS, where HAUS is the category of Hausdorff spaces with continuous maps as morphisms) by Porter in [28].

The last two sections treat Question 1.3 in the particular case when $\mathbf{D}$ is the subcategory of $\mathbf{C}$ whose objects are those isomorphic to a given object $X$. Particular study of Question 1.3 is given in the category of groups.

\section{Reflective subcategories and orthogonality}

Let us first fix some notations which will be used throughout this section. The symbol $\mathbf{C}$ will always denote a category, $\mathbf{D}$ will denote a full reflective subcategory of $\mathbf{C}$ which is isomorphism-closed. We also denote by $\mathbf{F}$ a left adjoint functor of the inclusion functor $\mathbf{I}: \mathbf{D} \rightarrow \mathbf{C}$ and $\mu$ the unit of the adjunction (for precise definitions, see [25]).

Let $\theta_{1}: A \rightarrow B, \theta_{2}: B \rightarrow C$ be two morphisms of $\mathbf{C}$ and $\theta_{3}=\theta_{2} \circ \theta_{1}$. Since hom $(-, X)$ is a contravariant functor, then

$$
\operatorname{hom}_{\mathbf{C}}\left(\theta_{3}, X\right)=\operatorname{hom}_{\mathbf{C}}\left(\theta_{1}, X\right) \circ \operatorname{hom}_{\mathbf{C}}\left(\theta_{2}, X\right) \text {. }
$$


Thus, if two of the three maps $\operatorname{hom}_{\mathbf{C}}\left(\theta_{1}, X\right)$, $\operatorname{hom}_{\mathbf{C}}\left(\theta_{2}, X\right)$, hom $\mathbf{C}\left(\theta_{3}, X\right)$ are bijective, then so is the third one. This leads to the following proposition.

Proposition 2.1 (never two without three). Let $A \stackrel{\theta_{1}}{\rightarrow} B \stackrel{\theta_{2}}{\rightarrow} C$ be two morphisms in $\mathbf{C}$ and $\theta_{3}=\theta_{2} \circ \theta_{1}$. If two of the morphisms $\theta_{1}, \theta_{2}$, and $\theta_{3}$ are in $\mathbf{D}^{\perp}$, then so is the third one.

Proposition 2.2. For each object $A$ in $\mathbf{C}$, the following statements are equivalent:

(i) $A$ is in $\mathbf{D}$;

(ii) $\mu_{A}: A \rightarrow \mathbf{F}(A)$ is an isomorphism.

The following result is an easy observation from [6].

Proposition 2.3. Let $f: A \rightarrow B$ be a morphism in $\mathrm{C}$. Then the following statements are equivalent:

(i) $f \in \mathbf{D}^{\perp}$;

(ii) $\mathbf{F}(f): \mathbf{F}(A) \rightarrow \mathbf{F}(B)$ is an isomorphism;

(iii) $F(f) \in \mathbf{D}^{\perp}$.

Let $F: \mathbf{C} \rightarrow \mathbf{C}^{\prime}$ be a functor. The class of morphisms of $\mathbf{C}$ rendered invertible by $F$ is sometimes denoted by $\Sigma_{F}[6]$ or $\mathscr{S}(F)$ [4]. Hence, Proposition 2.2 says exactly that $\mathbf{D}^{\perp}=\mathscr{S}(F)$.

Using Propositions 2.2 and 2.3, one may check easily the following.

Proposition 2.4. Let $f: A \rightarrow B$ be in $\mathbf{D}^{\perp}$. Then the following properties hold.

(1) If $A$ is an object of $\mathbf{D}$, then $f$ has a retraction $g: B \rightarrow A$ which is in $\mathbf{D}^{\perp}$ (in particular, $f$ is a monomorphism).

(2) If $A$ and $B$ are objects in $\mathbf{D}$, then $f$ is an isomorphism.

Remark 2.5. It is possible to have an arrow $f: A \rightarrow B$ in $\mathbf{D}^{\perp}$ such that $B$ is in $\mathbf{D}$, but $f$ has neither section nor retraction (see Example 3.4).

An affirmative answer to Question 1.2 is already in [19]; this yields the following.

Proposition 2.6. $\mathbf{D}^{\perp \perp}=\mathbf{D}$.

Proof. Clearly, $\mathbf{D}$ is contained in $\mathrm{D}^{\perp \perp}$.

Conversely, let $C$ be an object of $C$ lying in $\mathbf{D}^{\perp \perp}$. Since $\mu_{C}$ is in $\mathbf{D}^{\perp}$, there exists a unique morphism $g: \mathrm{F}(C) \rightarrow C$ such that the diagram

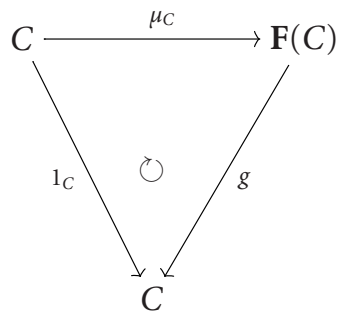

is commutative. 
3390 The envelope of a subcategory in topology and group theory

Thus, the diagram

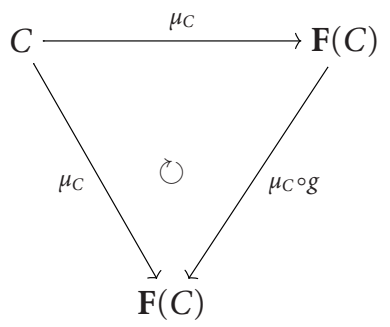

commutes.

Since, in addition, $\mu_{C} \in \mathbf{D}^{\perp}$, we get $\mu_{C} \circ g=1_{\mathbf{F}(C)}$. Therefore, $\mu_{C}$ is an isomorphism, and consequently $C$ is a $\mathbf{D}$-object by Proposition 2.2.

Proposition 2.7. Let $\mathbf{D}_{1}$ and $\mathbf{D}_{2}$ be two full reflective isomorphism-closed subcategories of a category $\mathbf{C}$. For $i \in\{1,2\}$, denote by $\mathbf{F}_{i}$ a left adjoint functor of the inclusion functor from $\mathbf{D}_{i}$ into $\mathbf{C}$.

Then the following statements are equivalent:

(i) $\mathbf{D}_{1}=\mathbf{D}_{2}$;

(ii) $\mathbf{F}_{1}$ and $\mathbf{F}_{2}$ are naturally isomorphic;

(iii) for each objects $A, B$ in $\mathbf{C}$, hom $\mathbf{C}(A, B) \cap \mathbf{D}_{1}{ }^{\perp}=\operatorname{hom}_{\mathbf{C}}(A, B) \cap \mathbf{D}_{\mathbf{2}}{ }^{\perp}$.

Proof. (i) $\Rightarrow$ (ii). It is well known that any two left adjoint functors for a given functor are naturally isomorphic [25].

(ii) $\Rightarrow$ (iii). Let $\eta$ be a natural isomorphism from $\mathbf{F}_{1}$ to $\mathbf{F}_{2}$ and $f: A \rightarrow B$ a morphism in C. Hence, the diagram

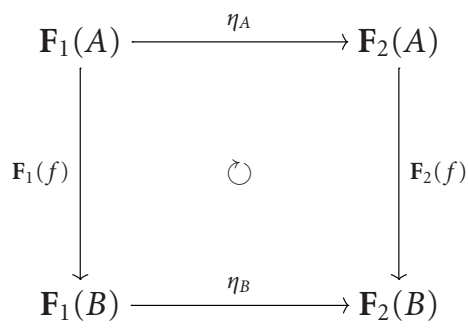

commutes. On the other hand, $\eta_{A}$ and $\eta_{B}$ are isomorphisms, and it follows that $\mathbf{F}_{1}(f)$ is an isomorphism if and only if so is $\mathbf{F}_{2}(f)$. Therefore, $\operatorname{hom}_{\mathbf{C}}(A, B) \cap \mathbf{D}_{\mathbf{1}}{ }^{\perp}=\operatorname{hom}_{\mathbf{C}}(A, B) \cap$ $\mathbf{D}_{2}{ }^{\perp}$, for each objects $A, B$ in $\mathbf{C}$ by Proposition 2.3 .

(iii) $\Rightarrow$ (i). The proof follows immediately from Proposition 2.6.

The following result gives more information about reflective subcategories.

Proposition 2.8. Let $\mathbf{D}$ be a subcategory of a category $\mathbf{C}$ and $\mathbf{F}: \mathbf{C} \rightarrow \mathbf{D}$ a covariant functor. Then the following statements are equivalent:

(1) $\mathbf{F}$ is a left adjoint functor of the inclusion functor $\mathbf{I}: \mathbf{D} \rightarrow \mathbf{C}$;

(2) there exists a natural transformation $\mu: 1_{\mathbf{C}} \rightarrow \mathbf{I} \circ \mathbf{F}$ such that the following properties 
hold:

(i) for each object $A$ in $\mathbf{C}, \mathbf{F}\left(\mu_{A}\right)$ is an isomorphism,

(ii) for each object $A$ in $\mathbf{D}, \mu_{A}$ is an isomorphism.

Proof. (1) $\Rightarrow(2)$. Statement (1) is equivalent to $\mathbf{D}$ being a reflective subcategory of $\mathbf{C}$. Now, since $\mu_{A}$ is in $\mathbf{D}^{\perp}$, we conclude that $\mathbf{F}\left(\mu_{A}\right)$ is an isomorphism, by Proposition 2.3.

If $A$ is in $\mathbf{D}$, then, according to Proposition 2.2, $\mu_{A}$ is an isomorphism.

$(2) \Rightarrow(1)$. We are aiming to prove that $\left(\mathbf{F}(A), \mu_{A}\right)$ is a universal to the inclusion functor I : $\mathbf{D} \rightarrow \mathbf{C}$ from $A$.

Let $C$ be an object of $\mathbf{D}$ and $f: A \rightarrow C$ a morphism in $\mathbf{C}$. We must prove that there is a unique morphism $\tilde{f}: \mathbf{F}(A) \rightarrow C$ such that $\tilde{f} \circ \mu_{A}=f$.

Suppose that such a morphism $\tilde{f}$ exists. Then we have $\mathbf{F}(\tilde{f}) \circ \mathbf{F}\left(\mu_{A}\right)=\mathbf{F}(f)$. Thus $\mathbf{F}(\tilde{f})=F(f) \circ\left(\mathbf{F}\left(\mu_{A}\right)\right)^{-1}$.

On the other hand, the diagram

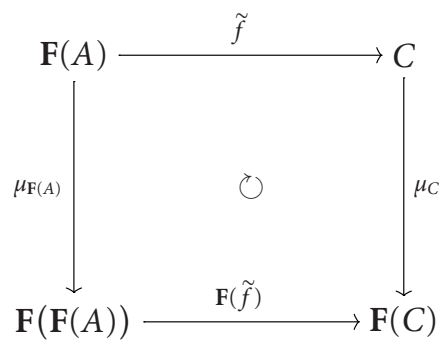

commutes. Consequently,

$$
\tilde{f}=\left(\mu_{C}\right)^{-1} \circ \mathbf{F}(\tilde{f}) \circ \mu_{\mathbf{F}(A)}=\left(\mu_{C}\right)^{-1} \circ \mathbf{F}(f) \circ\left(\mathbf{F}\left(\mu_{A}\right)\right)^{-1} \circ \mu_{\mathbf{F}(A)} .
$$

This implies the uniqueness of $\tilde{f}$, if it exists. Now, it suffices to verify that $\tilde{f}=\left(\mu_{C}\right)^{-1}$ 。 $\mathbf{F}(f) \circ\left(\mathbf{F}\left(\mu_{A}\right)\right)^{-1} \circ \mu_{\mathbf{F}(A)}$ does the job. Indeed, the following diagrams

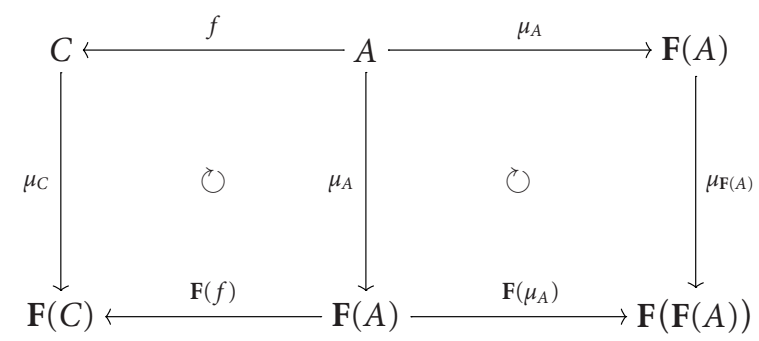

are commutative. Hence,

$$
\begin{aligned}
\tilde{f} \circ \mu_{A} & =\left(\mu_{C}\right)^{-1} \circ \mathbf{F}(f) \circ\left(\mathbf{F}\left(\mu_{A}\right)\right)^{-1} \circ \mu_{\mathbf{F}(A)} \circ \mu_{A} \\
& =\left(\mu_{C}\right)^{-1} \circ \mathbf{F}(f) \circ\left(\mathbf{F}\left(\mu_{A}\right)\right)^{-1} \circ \mathbf{F}\left(\mu_{A}\right) \circ \mu_{A} \\
& =\left(\mu_{C}\right)^{-1} \circ \mathbf{F}(f) \circ \mu_{A}=\left(\mu_{C}\right)^{-1} \circ \mu_{C} \circ f=f .
\end{aligned}
$$




\section{An example of orthogonality class of morphisms}

Let $X$ be a topological space, we denote by $\mathfrak{O}(X)$ the set of all open subsets of $X$. Recall that a continuous map $g: Y \rightarrow Z$ is said to be a quasihomeomorphism if $U \mapsto g^{-1}(U)$ defines a bijection $\mathfrak{O}(Z) \rightarrow \mathfrak{O}(Y)$.

Recall that a subset of a topological space $X$ is said to be locally closed if it is the intersection of an open subset and a closed subset of $X$. A subset $S$ of a topological space $X$ is said to be strongly dense in $X$ if $S$ meets every nonempty locally closed subset of $X$. Thus, a subset $S$ of $X$ is strongly dense if and only if the canonical injection $S \hookrightarrow X$ is a quasihomeomorphism. It is well known that a continuous map $q: X \rightarrow Y$ is a quasihomeomorphism if and only if the topology of $X$ is the inverse image by $q$ of that of $Y$ and the subset $q(X)$ is strongly dense in $Y[16]$.

The notion of quasihomeomorphism is used in algebraic geometry. It has been recently shown that this notion arises naturally in the theory of some foliations associated to closed connected manifolds (see $[2,3]$ ). It is worth noting that quasihomeomorphisms are also linked with sober spaces.

A subset $Y$ of a topological space $X$ is said to be irreducible if each nonempty open subset of $Y$ is dense in $Y$. Let $C$ be a closed subset of $X$. We say that $C$ has a generic point if there is $a \in C$ such that $C=\overline{\{a\}}$. Recall that a topological space $X$ is said to be sober if any nonempty irreducible closed subset of $X$ has a unique generic point. Let $X$ be a topological space and $\mathbf{S}(X)$ the set of all irreducible closed subsets of $X$ [16]. Let $U$ be an open subset of $X$, set $\tilde{U}=\{F \in \mathbf{S}(X) \mid U \cap F \neq \varnothing\}$, then the collection $\{\tilde{U} \mid U \in \mathfrak{O}(X)\}$ provides a topology on $\mathbf{S}(X)$ and the following properties hold.

(i) The map $\eta_{X}: X \rightarrow \mathbf{S}(X)$ defined by $\eta_{X}(x)=\overline{\{x\}}$ is a quasihomeomorphism.

(ii) $\mathbf{S}(X)$ is a sober space.

(iii) The topological space $\mathbf{S}(X)$ is called the soberification of $X$, and the assignment $X \rightarrow \mathbf{S}(X)$ defines a functor from the category of topological spaces TOP to TOP [16].

The soberification serves, sometime, to give topological characterization of particular spaces (see, e.g., [11]).

We denote by SOB the full subcategory of TOP whose objects are sober spaces. It is clear that $\eta$ is a natural transformation from the functor $1_{\text {TOP }}$ to the functor $\mathbf{I} \circ \mathbf{S}$, where I : SOB $\rightarrow$ TOP is the inclusion functor. Let $X$ and $Y$ be two topological spaces, we will denote $\mathbf{Q H}(X, Y)$ the set of all quasihomeomorphisms from $X$ to $Y$.

It is well known that SOB is a reflective full subcategory of TOP [16]. We are aiming to determine the orthogonality class of morphisms $\mathbf{S O B}^{\perp}$.

Notice that all the material of this section may be derived from $[1,12]$; and for the sake of completeness, we will give all the details.

First, notice that Proposition 3.1 follows from well-established results in frame (or locale) theory. The adjunction

$$
\Omega \vdash \Sigma: \text { FRAME } \longrightarrow \text { TOP }
$$

where $\Omega$ is the open set functor, describes the soberification reflector by $\Sigma \circ \Omega$. The 
quasihomeomorphisms are exactly those maps inverted by the reflector (see, e.g., Johnstone [23]).

Proposition 3.1. The orthogonality class $\mathbf{S O B}^{\perp}$ of TOP is the class of all quasihomeomorphisms.

We need a technical lemma.

Lemma 3.2. Let $q: X \rightarrow Y$ be a quasihomeomorphism. Then the following properties hold.

(1) If $X$ is a $T_{0}$-space, then $q$ is one-to-one.

(2) If $X$ is sober and $Y$ is a $T_{0}$-space, then $q$ is a homeomorphism.

Proof. (1) Let $x_{1}, x_{2}$ be two points of $X$ with $q\left(x_{1}\right)=q\left(x_{2}\right)$. Suppose that $x_{1} \neq x_{2}$, then there exists an open subset $U$ of $X$ such that $x_{1} \in U$ and $x_{2} \notin U$. Since there exists an open subset $V$ of $Y$ satisfying $q^{-1}(V)=U$, we get $q\left(x_{1}\right) \in V$ and $q\left(x_{2}\right) \notin V$, which is impossible. It follows that $q$ is one-to-one.

(2) (i) We start with the obvious observation that if $S$ is a closed subset of $Y$, then $S$ is irreducible if and only if so is $q^{-1}(S)$.

(ii) Let us prove that $q$ is surjective. For this end, let $y \in Y$, according to the above observation, $q^{-1}(\overline{\{y\}})$ is a nonempty irreducible closed subset of $X$. Hence, $q^{-1}(\overline{\{y\}})$ has a generic point $x$. Thus, we have the containments

$$
\overline{\{x\}} \subseteq q^{-1}(\overline{\{q(x)\}}) \subseteq q^{-1}(\overline{\{y\}})=\overline{\{x\}}
$$

Then, $q^{-1}(\overline{\{q(x)\}})=q^{-1}(\overline{\{y\}})$. It follows, from the fact that $q$ is a quasihomeomorphism, that $\overline{\{q(x)\}}=\overline{\{y\}}$. Since $Y$ is a $T_{0}$-space, we get $q(x)=y$. This proves that $q$ is a surjective map, and thus $q$ is bijective. One may easily see that bijective quasihomeomorphisms are homeomorphisms.

Proof of Proposition 3.1. According to Proposition 2.3, one is brought back to prove that for each topological spaces $X, Y$ and each continuous map $q: X \rightarrow Y$, the following statements are equivalent:

(a) $q$ is a quasihomeomorphism;

(b) $\mathbf{S}(q)$ is a homeomorphism.

Remark that the diagram

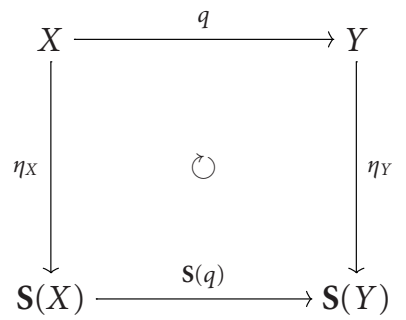

is commutative. 
3394 The envelope of a subcategory in topology and group theory

(a) $\Rightarrow(\mathrm{b})$. Since $\eta_{Y} \circ q=\mathbf{S}(q) \circ \eta_{X}$ is a quasihomeomorphism, the map $\mathbf{S}(q)$ is necessarily a quasihomeomorphism. Thus, following Lemma 3.2, $\mathbf{S}(q)$ is a homeomorphism.

(b) $\Rightarrow(\mathrm{a})$. Since $\eta_{X}=\left((\mathbf{S}(q))^{-1} \circ \eta_{Y}\right) \circ q$ and $(\mathbf{S}(q))^{-1} \circ \eta_{Y}$ are quasihomeomorphisms, it is easily seen that $q$ is a quasihomeomorphism.

Corollary 3.3. (1) Let $Z$ be a topological space. Then the following conditions are equivalent.

(i) $Z$ is a sober space.

(ii) For each quasihomeomorphism $g: Y \rightarrow X$ and each continuous map $f: Y \rightarrow Z$, there exists one and only one continuous map $F: X \rightarrow Z$ such that $F \circ g=f$.

(2) Let $g: Y \rightarrow X$ be a continuous map. Then the following conditions are equivalent:

(i) $g$ is a quasihomeomorphism;

(ii) for each sober space $Z$ and each continuous map $f: Y \rightarrow Z$, there exists one and only one continuous map $F: X \rightarrow Z$ such that $F \circ g=f$.

Now, we are in a position to give an example promised in Remark 2.5.

Example 3.4. Let $X$ be a $T_{0}$-space which is not sober. Then $\eta_{X}: X \rightarrow \mathbf{S}(X)$ is a quasihomeomorphism which is not a homeomorphism.

The mapping $\eta_{X}$ is in $\mathbf{S O B}^{\perp}$ and $\mathbf{S}(X)$ is an object of the reflective subcategory $\mathbf{S O B}$ of TOP, but $\eta_{X}$ has neither section nor retraction. Indeed, suppose that the following hold.

(i) Suppose that there exists a continuous map $g: \mathbf{S}(X) \rightarrow X$ such that $g \circ \eta_{X}=1_{X}$. Then $\left(\eta_{X} \circ g\right) \circ \eta_{X}=\eta_{X}$. This is to say that the diagram

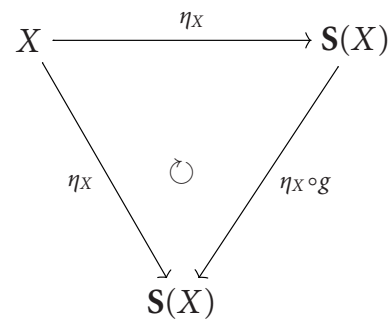

commutes.

Since $\mathbf{S}(X)$ is sober and $\eta_{X}$ is orthogonal to $\mathbf{S O B}$, we must have $\eta_{X} \circ g=1_{\mathbf{S}(X)}$. It follows that $\eta_{X}$ is a homeomorphism, a contradiction.

(ii) Suppose that there exists a continuous map $g: \mathbf{S}(X) \rightarrow X$ such that $\eta_{X} \circ g=1_{\mathbf{S}}(X)$. Then $\eta_{X}$ is a surjective quasihomeomorphism. Thus $\eta_{X}$ is a bijective quasihomeomorphism. Hence $\eta_{X}$ is a homeomorphism, a contradiction.

\section{The envelope of a subcategory}

This section is devoted to answer Question 1.3.

Let $\mathbf{C}$ be a category and let $\mathbf{D}$ be a subcategory (no longer reflective and not assumed to be isomorphism closed). 
4.1. Definitions and remarks. Let $\mathbf{C}$ be a category and $X$ an object of $\mathbf{C}$. By a $\mathbf{D}$-ification of $X$, we mean a morphism $p: X \rightarrow X^{\prime}$ such that $X^{\prime}$ is an object of $\mathbf{D}$ and $p$ is orthogonal to $\mathbf{D}$. The object $X$ is said to be $\mathbf{D}$-ifiable if it has a $\mathbf{D}$-ification. We denote by $\operatorname{Env}_{\mathbf{C}}(\mathbf{D})$ the full subcategory of $\mathbf{C}$ whose objects are the $\mathbf{D}$-ifiable objects of $\mathbf{C}$, this subcategory will be called the envelope of $\mathbf{D}$ in $\mathbf{C}$.

Remarks 4.1. (1) D is a subcategory of $\operatorname{Env}_{\mathbf{C}}(\mathbf{D})$ and $\operatorname{Env}_{\mathbf{C}}(\mathbf{D})$ is isomorphism-closed.

(2) $\mathbf{D}$ is a reflective subcategory of $\operatorname{Env}_{\mathbf{C}}(\mathrm{D})$.

(3) $\operatorname{Env}_{\mathbf{C}}(\mathbf{D})$ is the largest subcategory of $\mathbf{C}$ in which $\mathbf{D}$ is a reflective subcategory.

(4) $\operatorname{Env}_{\mathbf{C}}\left(\operatorname{Env}_{\mathbf{C}}(\mathbf{D})\right)=\operatorname{Env}_{\mathbf{C}}(\mathbf{D})$.

(5) If $\mathbf{D}$ is a subcategory of $\mathbf{C}_{1}$ and $\mathbf{C}_{1}$ is a subcategory of $\mathbf{C}$, then $\operatorname{Env}_{\mathbf{C}}\left(\operatorname{Env}_{\mathbf{C}_{1}}(\mathbf{D})\right)=$ $\operatorname{Env}_{\mathbf{C}}(\mathbf{D})$.

The following result is equivalent to Remark 4.1(5). This result facilitates verification of the fact that some objects are D-ifiable.

Proposition 4.2. Let $\mathbf{D}$ be a subcategory of $\mathbf{C}_{1}$ and $\mathbf{C}_{1}$ a subcategory of $\mathbf{C}$. Suppose that $X^{\prime}$ is a $\mathbf{C}_{1}$-ification of the object $X$ of $\mathbf{C}$. Then the following statements are equivalent:

(i) $X$ is D-ifiable;

(ii) $X^{\prime}$ is D-ifiable.

Example 4.3. Let $n \in\{0,1,2,3\}$, TOP ${ }_{n}$ denotes the full subcategory of all $T_{n}$-spaces in TOP, with $T_{4}=$ Normal, $T_{3}=$ Regular. Then $\operatorname{Env}_{\mathbf{T O P}_{n}}\left(\mathbf{T O P}_{n+1}\right)=\mathbf{T O P}_{n}$, and thus every topological space is $\mathbf{T O P}_{n}$-ifiable.

4.2. Spectralifiable spaces. Let $\operatorname{Spec}(R)$ denote the set of prime ideals of a commutative ring with identity $R$. Recall that the Zariski topology for $\operatorname{Spec}(R)$ is defined by letting $C \subseteq$ $\operatorname{Spec}(R)$ be closed if and only if there exists an ideal $\mathfrak{a}$ of $R$ such that $C=\{\mathfrak{p} \in \operatorname{Spec}(R) \mid$ $\mathfrak{p} \supseteq \mathfrak{a}\}$. The topological question of characterizing spectral spaces (i.e., topological spaces homeomorphic to the prime spectrum of a ring equipped with the Zariski topology) was completely answered by Hochster in [21]: a topology $\mathscr{T}$ on a set $X$ is spectral if and only if the following axioms hold.

(i) $X$ is a sober space.

(ii) $X$ is compact and has a basis of compact open subsets.

(iii) The family of compact open subsets of $X$ is closed under finite intersections.

Following Hochster [21], a continuous map of spectral spaces is said to be spectral if inverse images of compact open sets are compact. Let $\mathscr{S}$ be the subcategory of TOP consisting of spectral spaces and spectral maps. Hochster has extended the notion of spectral map to a larger class of spaces. Call a space semispectral if the intersection of any two compact open subsets is compact. Call an open subset $U$ of a topological space $X$ intersection compact open, or ICO, if for every compact open set $Q$ of $X, U \cap Q$ is compact. Thus, $X$ is semispectral if and only if the compact open sets are ICO. Then a continuous map $f$ of semispectral spaces will be called spectral if $f^{-1}$ carries ICO sets to ICO sets. The semispectral spaces and spectral maps form a category $\mathfrak{U}$. It is easily seen that $\mathscr{Y}$ is a full subcategory of $\mathfrak{U}$.

In the same paper [21], Hochster has introduced the notion of spectralifiable space. By a spectralification of a semispectral space $X$, we mean a spectral embedding $g$ of $X$ 
into a spectral space $X^{\prime}$ such that for every spectral space $Y$ and spectral map $f$ from $X$ to $Y$, there is a unique spectral map $f^{\prime}$ from $X^{\prime}$ to $Y$ such that $f=f^{\prime} \circ g$. The space $X$ is said to be spectralifiable if it has a spectralification [21]. When a semispectral space is spectralifible, we will say that it is $H$-spectralifiable.

A complete characterization of $H$-spectralifiable spaces is given by Hochster in the following.

Theorem 4.4 (see Hochster [21]). Let X be a semispectral space. Then the following conditions are equivalent:

(i) $X$ is $H$-spectralifiable;

(ii) $X$ is $T_{0}$ and the ICO sets are an open basis;

(iii) $X$ can be spectrally embedded in some spectral space.

Problem 4.5. It is clear that every $H$-spectralifiable space of $\mathfrak{U}$ is $\mathscr{Y}$-ifiable. Is the converse true? what are $\mathscr{S}$-ifiable spaces of $\mathfrak{U}$ ?

4.3. Jacspectralifiable spaces. Recall that a topological space $X$ is said to be a Jacobson space if the set $\mathscr{C}(X)$ of all closed points of $X$ is strongly dense in $X$ [16]. Obviously, when $X$ is a topological space, $\operatorname{Jac}(X)=\{x \in X \mid \overline{\{x\}}=\overline{\overline{\{x\}} \cap \mathscr{C}(X)}\}$ is a Jacobson space; we call it the Jacobson subspace of $X$. Clearly, $\operatorname{Jac}(X)$ is the largest subset of $X$ in which $\mathscr{b}(X)$ is strongly dense. Hence, the canonical injection $\mathscr{C}(X) \hookrightarrow \operatorname{Jac}(X)$ is a quasihomeomorphism.

Let $R$ be a ring, we denote by $\operatorname{Jac}(R)$ the Jacobson subspace of $\operatorname{Spec}(R)$. It is easily seen that a prime ideal $\mathfrak{p}$ of $R$ is in $\operatorname{Jac}(R)$ if and only if $\mathfrak{p}$ is the intersection of all maximal ideals $\mathfrak{m}$ of $R$ such that $\mathfrak{p} \subseteq \mathfrak{m}$. A jacspectral space is defined to be a topological space homeomorphic to the Jacobson space of $\operatorname{Spec}(R)$ for some $\operatorname{ring} R$.

In [1], Bouacida et al. have given a nice topological characterization of jacspectral spaces. For the sake of completeness, we will prove this result but with some changes in the proof.

We need a lemma, its proof is obvious, and therefore it is omitted.

Lemma 4.6. Let $q: X \rightarrow Y$ be a quasihomeomorphism.

(1) If $U$ is an open subset of $Y$, then the following statements are equivalent:

(i) $U$ is compact;

(ii) $q^{-1}(U)$ is compact.

(2) If $q$ is injective, then $\mathscr{C}(Y) \subseteq q(\mathscr{C}(X))$.

(3) Let $X$ be a $T_{0}$-space. Then $q(\mathscr{C}(X))=\mathscr{C}(\mathbf{S}(X))$, where $q: X \rightarrow \mathbf{S}(X)$ is the injection of $X$ onto its soberification $\mathbf{S}(X)$.

(4) Let $S$ be a subset of $X$. Then the following statements are equivalent:

(i) $S$ is strongly dense in $X$;

(ii) $q(S)$ is strongly dense in $Y$.

(5) If $X$ is a $T_{0}$-space, then $X$ is Jacobson if and only if so is $\mathbf{S}(X)$.

We now head towards an important result which completely characterizes jacspectral spaces. 
Theorem 4.7. Let $X$ be a topological space. The following statements are equivalent:

(i) $X$ is a jacspectral space;

(ii) $X$ is a compact Jacobson sober space.

Proof. (i) $\Rightarrow$ (ii). Let $R$ be a ring and $X=\operatorname{Jac}(R)=\operatorname{Jac}(\operatorname{Spec}(R))$ the Jacobson space of $\operatorname{Spec}(R)$; then $X$ is a Jacobson space. One may check easily that $\operatorname{Jac}(R)$ is the soberification of $\operatorname{Max}(R)$ (the set of all maximal ideals of $R$ ). Thus $\operatorname{Jac}(R)$ is a sober space. On the other hand, $\operatorname{Max}(R)$ is compact by Hochster [21]. Moreover, the canonical injection $\operatorname{Max}(R) \hookrightarrow \operatorname{Jac}(R)$ is a quasihomeomorphism. Hence $X$ is compact by Lemma 4.6.

(ii) $\Rightarrow(\mathrm{i})$. Suppose that $X$ is a compact Jacobson sober space. We know that the canonical injection $\mathscr{C}(X) \hookrightarrow X$ is a quasihomeomorphism, whence $\mathscr{C}(X)$ is compact by Lemma 4.6. It follows that $\mathscr{C}(X)$ is a compact $T_{1}$-space. Therefore, there exists some ring $R$ such that $\mathscr{C}(X)$ is homeomorphic to $\operatorname{Max}(R)$ (see Hochster [21, Proposition 11]). Let $\varphi$ : $\mathscr{C}(X) \rightarrow \operatorname{Max}(R)$ be a homeomorphism and $i: \operatorname{Max}(R) \rightarrow \operatorname{Jac}(R)$ the canonical injection; then $f=i \circ \varphi: \mathscr{C}(X) \rightarrow \operatorname{Jac}(R)$ is a quasihomeomorphism. In view of Corollary 3.3, there exists a continuous extension $\tilde{f}: X \rightarrow \operatorname{Jac}(R)$. This extension is also a quasihomeomorphism. Now, since $X$ and $\operatorname{Jac}(R)$ are sober, $\tilde{f}$ is a homeomorphism by Proposition $2.4(2)$.

Let $\mathscr{I} \mathscr{S}$ be the full subcategory of TOP whose objects are jacspectral spaces. By a jacspectralifiable space, we mean a $\mathscr{\mathscr { S }} \mathscr{Y}$-ifiable topological space. Next, we give some examples of jacspectralifiable spaces.

Proposition 4.8. Let $X$ be a topological space. If the $T_{0}$-identification $\mathbf{T}_{0}(X)$ of $X$ is a Jacobson compact space, then $X$ is jacspectralifiable.

Proof. Following Proposition 4.2, it suffices to prove that each $T_{0}$ compact Jacobson space is jacspectralifiable (since each topological space is $\mathbf{T O P}_{0}$-ifiable).

Lemma 4.6 assures that the soberification $\mathbf{S}(X)$ is a compact Jacobson sober space. Hence, $S(X)$ is a jacspectral space, by Theorem 4.7. Now, Corollary 3.3 tells us that the canonical injection of $X$ into its soberification $\mathbf{S}(X)$ is a $\mathscr{I} \mathscr{Y}$-ification of $X$.

We state a similar problem to Problem 4.5.

Problem 4.9. What are jacspectralifiable spaces?

4.4. COMP-ifiable spaces. Let COMP be the full subcategory of TOP consisting of compact topological spaces. It is well known that a completely regular space is COMP-ifiable. The following is a more general result.

Let TOP $_{3.5}$ be the full subcategory of TOP consisting of completely regular spaces. It is known that every space has a $\mathbf{T O P}_{3.5}$-ification (see $\left.[26,27]\right)$. Now, if $F: \mathbf{A} \rightarrow \mathbf{B}$ and $G: \mathbf{B} \rightarrow \mathbf{C}$ are reflectors, then so is the composition $G \circ F$. This yields the following proposition.

Proposition 4.10. Every topological space is COMP-ifiable (i.e., COMP is reflective in TOP).

Let $f: X \rightarrow Y$ be a continuous map between completely regular spaces. A natural question is asked; when is the extension $\beta(f): \beta(X) \rightarrow \beta(Y)$ a homeomorphism? 
3398 The envelope of a subcategory in topology and group theory

According to the paper of Holgate [22], we get the following.

Proposition 4.11. Let $X$ and $Y$ be two completely regular spaces and $f: X \rightarrow Y$ a continuous map. Then $\beta(f): \beta(X) \rightarrow \beta(Y)$ is a homeomorphism if and only if $f$ is an embedding and $\beta(f(X))=\beta(Y)$.

Proof. First, suppose that $\beta(f)$ is a homeomorphism. Then, the restriction of $\beta(f)$ to $X$ is an embedding, which implies that $f: X \rightarrow Y$ is an embedding. Clearly, $f(X) \subseteq Y \subseteq \beta(Y)$ and

$$
X \subseteq \beta(f)^{-1}(Y) \subseteq \beta(X)
$$

Now, we have the following commutative diagram:

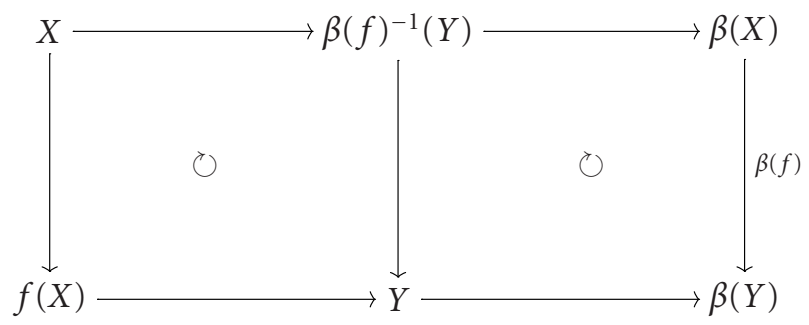

Since three natural maps indicated by downward arrows are homeomorphisms and $\beta\left(\beta(f)^{-1}(Y)\right)=\beta(X)$, we have $\beta(Y)=\beta(f(X))$. Conversely, if $f: X \rightarrow Y$ is an embedding, then the extension $\beta\left(f^{\prime}\right): \beta(X) \rightarrow \beta(f(X))$ is a homeomorphism. Hence, if $\beta(f(X))$ $=\beta(Y)$ in addition, then $\beta\left(f^{\prime}\right)=\beta(f)$, and hence $\beta(f): \beta(X) \rightarrow \beta(Y)$ is a homeomorphism.

\section{The $X$-ification}

5.1. Definitions and remarks. Let $X, Y$ be two objects of a category C. By an $X$-ification of $Y$, we mean a morphism $p: Y \rightarrow X$ such that $p$ is orthogonal to $X$. We say that $Y$ is $X$-ifiable if there is an $X$-ification of $Y$. This is a particular case when $\mathbf{D}$ is the subcategory of $\mathbf{C}$ whose objects are those isomorphic to a given object $X$.

Of course, if $Y$ is isomorphic to $X$, then $Y$ is $X$-ifiable. Also, if $Y$ is $X$-ifiable and $X$ is isomorphic to $Z$, then $Y$ is $Z$-ifiable.

The following result will be needed in order to discuss the notion of $X$-ifiable objects.

Proposition 5.1. Let $\mathbf{C}$ be a category and let $\mathbf{D}$ be a subcategory of $\mathbf{C}$. If $X$ is in $\mathbf{D}$ and $Z$ is a D-ification of $Y$, then the following statements are equivalent:

(i) $Y$ is $X$-ifiable;

(ii) $Z$ is $X$-ifiable.

Proof. (i) $\Rightarrow$ (ii). Let $p: Y \rightarrow X$ be an $X$-ification of $Y$ and $q: Y \rightarrow Z$ a D-ification of $Y$. Since $q \perp \mathbf{D}$, there is a unique morphism $\tilde{p}: Z \rightarrow X$ such that $\tilde{p} \circ q=p$. It is easily seen that $\tilde{p}: Z \rightarrow X$ is an $X$-ification of $Z$. 
(ii) $\Rightarrow$ (i). One may check easily that if $p: Y \rightarrow Z$ is a D-ification of $Y$ and $q: Z \rightarrow X$ is an $X$-ification of $Z$, then $q \circ p: Y \rightarrow X$ is an $X$-ification of $Y$.

5.2. $G$-ification in the category of groups. Let $G$ be a group. We turn our attention to the study of $G$-ifiable groups in the category of groups GRP.

Examples 5.2. (1) Let (0) be the trivial group. Then each group is (0)-ifiable.

(2) If $G$ is a cyclic group, then $\mathbb{Z}$ is $G$-ifiable. It suffices to remark that $p: \mathbb{Z} \rightarrow G=\mathbb{Z} \theta$, defined by $n \mapsto n \theta$, is a $G$-ification of $\mathbb{Z}$.

Remark 5.3. Let $\mathrm{AB}$ be the full subcategory of GRP whose objects are the Abelian groups. It is well known that $\mathrm{AB}$ is a reflective subcategory of GRP. Thus following Proposition 5.1, if $G$ is an Abelian group and $H$ is a group, then $H$ is $G$-ifiable if and only if $H / D(H)$ is $G$-ifiable (where $D(H)$ is the commutator subgroup of $H$ ).

The following notion proves to be useful for the study of $G$-ifiable groups.

It is worth noting that the concept of zero or constant morphisms was first introduced and studied by Herrlich in [19], but has been considerably developed. In the algebraic context, much work has been done in considering the associated "torsion theories," see, for example, [10].

Definition 5.4. Let $G, H$ be two groups. Say that $H$ is $G$-terminal if each morphism from $G$ to $H$ is zero.

Example 5.5. For any torsion Abelian group $G$, the group $\mathbb{Z}$ is $G$-terminal.

Remarks 5.6. (1) Let $\left(G_{i}, i \in I\right)$ and $\left(H_{j}, j \in J\right)$ be two collections of groups such that each $H_{j}$ is $G_{i}$-terminal. Then, the direct product $\prod_{j \in J} H_{j}$ is $\prod_{i \in I} G_{i}$-terminal.

(2) If $H$ is $G$-terminal and $K$ a normal subgroup of $G$, then $H$ is terminal relative to the quotient group $G / K$.

The following result gives more examples of groups such that the group $\mathbb{Z}$ is terminal relative to them.

Proposition 5.7. Let $n \in \mathbb{N}-\{0,1\}$ and $G$ an $n$-divisible Abelian group (i.e., for each $x \in G$, there exists $y \in G$ such that $x=n y)$. Then $\mathbb{Z}$ is $G$-terminal.

Proof. Suppose that there exists a nonzero morphism of groups $p: G \rightarrow \mathbb{Z}$. Hence, there exists $a \in \mathbb{Z}-\{0\}$ such that $p(G)=a \mathbb{Z}$. Let $\theta \in G$ such that $p(\theta)=a$. It is easily seen that $G=\operatorname{Ker}(p) \oplus \mathbb{Z} \theta$.

Since $G$ is $n$-divisible, there exists $\theta_{1} \in G$ such that $\theta=n \theta_{1}$. We write $\theta_{1}=k \theta+x$, where $x \in \operatorname{Ker}(p)$ and $k \in \mathbb{Z}$. This gives $\theta=n(k \theta+x)=\theta+0$. By uniqueness of decomposition of $\theta$ in the direct sum $\mathbb{Z} \theta \oplus \operatorname{Ker}(p)$, we get $\theta=n k \theta$. Thus $a=n k a$; so that $1=n k$; and thus $n=1$, a contradiction.

Remark 5.8. It follows from Proposition 5.7 that $\mathbb{Z}$ is terminal relative to $\mathbb{Q}, \mathbb{R}, \mathbb{C}$.

The following observation gives some examples of $G$-ifiable groups.

Proposition 5.9. Let $G, H$ be two groups such that $G$ is $H$-terminal. Then the direct product $G \times H$ is a $G$-ifiable group. 
Proof. Let $p: G \times H \rightarrow G$ be the canonical projection. Let us verify that $p$ is a $G$-ification of $G \times H$. Let $f: G \times H \rightarrow G$ be a morphism. Since $G$ is $H$-terminal, $f(x, y)=f(x, 0)$, for each $x \in G$ and $y \in H$. Now, it is easily seen that $\tilde{f}: G \rightarrow G$ defined by $\tilde{f}(x)=f(x, 0)$ is the unique endomorphism of $G$ such that $\tilde{f} \circ p=f$. Thus $G \times H$ is $G$-ifiable.

The above proposition makes the following definition allowable.

Definition 5.10. Let $\mathbf{C}$ be a subcategory of GRP. A group $G$ is said to be a Tunisian group (T-group) of $\mathbf{C}$ if each $G$-ifiable group in $\mathbf{C}$ is isomorphic to a direct product $G \times H$, where $H$ is a group such that $G$ is $H$-terminal.

Of course, the trivial group (0) is a T-group in GRP.

The following remark will be useful in the next result.

Remark 5.11. Let $H$ be a nontrivial group and let $p: G \rightarrow H$ be an $H$-ification of a group $G$. Then $p$ is a nonzero morphism of groups. Indeed, if it is not the case, then $1_{H} \circ p=$ $\theta \circ p$ would imply that $\theta=1_{H}$ (where $\theta: H \rightarrow H$ is the zero morphism), and thus $H$ would be the trivial group ( 0 ), a contradiction. Therefore, the $H$-ification of $G$ is nonzero.

THEOREM 5.12. Let $\mathcal{H}$ be the full subcategory of GRP whose objects are the groups such that every subgroup is normal. Then $\mathbb{Z}$ is a $\mathbf{T}$-group in $\mathcal{H}$.

Proof. Let $(G,+)$ be a $\mathbb{Z}$-ifiable group in $\mathscr{H}$. Let $p: G \rightarrow \mathbb{Z}$ be a $\mathbb{Z}$-ification of $G$. By Remark 5.11, $p$ is a nonzero morphism of groups. Hence, there exists $a \in \mathbb{Z}-\{0\}$ such that $p(G)=\mathbb{Z} a$.

Let $x_{0} \in G$ such that $p\left(x_{0}\right)=a$. It is an easy exercise to verify that $G=\operatorname{Ker}(p) \oplus \mathbb{Z} x_{0}$. Now, since $x_{0}$ has infinite order, we get easily $G \sim \mathbb{Z} \times \operatorname{Ker}(p)$. It remains to prove that $\mathbb{Z}$ is $\operatorname{Ker}(p)$-terminal. Let $g: \operatorname{Ker}(p) \rightarrow \mathbb{Z}$ be a morphism. Consider the morphism $f: G=$ $\operatorname{Ker}(p) \oplus \mathbb{Z} x_{0} \rightarrow \mathbb{Z}$ defined by $f(\alpha+\beta)=g(\alpha)$, for each $(\alpha, \beta) \in \operatorname{Ker}(p) \times \mathbb{Z} x_{0}$ (note that one does not need the commutativity to verify that $f$ is a morphism, since if $G=H \oplus K$, with $H, K$ normal subgroups of $G$, then $h+k=k+h$ for each $(h, k) \in H \times K)$.

Since $p: G \rightarrow \mathbb{Z}$ is a $\mathbb{Z}$-ification of $G$, there exists a unique morphism $\tilde{f}: \mathbb{Z} \rightarrow \mathbb{Z}$ such that $\tilde{f} \circ p=f$. Therefore, $g(x)=0$, for each $x \in \operatorname{Ker}(p)$. This proves that $\mathbb{Z}$ is $\operatorname{Ker}(p)$ terminal, showing that $\mathbb{Z}$ is a $\mathbf{T}$-group in the category $\mathcal{H}$.

Remark 5.13. The quotient of a T-group need not be a T-group (see Corollary 6.10).

\section{Examples of non-T-groups}

Everything that we discuss here is essentially made to exhibit non-T-groups of arbitrary orders. We also prove that the quotient of a T-group need not be a T-group.

The following definition is natural, besides it will prove extremely useful in the study of morphisms from $\mathbb{Z} / n \mathbb{Z}$ to $\mathbb{Z} / m \mathbb{Z}$.

Definition 6.1. Two groups $G, H$ are said to be relatively terminal if $G$ is $H$-terminal and $H$ is $G$-terminal.

Proposition 6.2. Let $G, H$ be two finite groups such that $\operatorname{gcd}(o(G), o(H))=1$, where $o(G)$ denotes the order of $G$. Then $G, H$ are relatively terminal. 
Proof. It suffices to prove that $H$ is $G$-terminal. For this, consider $p \in \operatorname{hom}(G, H)$ and $x \in G$, we have to prove that $p(x)=0_{H}$. Since $o(G) x=0_{G}$, we have $o(G) p(x)=0_{H}$. Hence, $o(p(x))$ divides $o(G)$. Thus, $o(p(x))$ divides $\operatorname{gcd}(o(G), o(H))=1$. Therefore, $o(p(x))=1$, so that $p(x)=0$.

Before stating our next result, let us recall some elementary facts about congruences.

Remark 6.3. (1) Cancelation law. Let $a, b, c, m$ be in $\mathbb{Z}$ such that $c \neq 0$ and let $d=\operatorname{gcd}(m, c)$. Then $a c \equiv b c(\bmod m)$ if and only if $a \equiv b(\bmod (m / d))$.

(2) Let $\alpha \in \mathbb{Z}$. Then $\operatorname{gcd}(\alpha, m)=1$ if and only if there exists $\beta \in \mathbb{Z}$ such that $\alpha \beta \equiv 1$ $(\bmod m)$.

Proposition 6.4. Let $m, n \in \mathbb{N}$ be such that one of them is nonzero. Then, (hom $(\mathbb{Z} / n \mathbb{Z}$, $\mathbb{Z} / m \mathbb{Z}),+$ ) is a cyclic group of order $d=\operatorname{gcd}(m, n)$.

Proof. (i) First, suppose that $d=1$. According to Proposition $6.2, \mathbb{Z} / n \mathbb{Z}$ and $\mathbb{Z} / m \mathbb{Z}$ are relatively terminal. Hence, hom $(\mathbb{Z} / n \mathbb{Z}, \mathbb{Z} / m \mathbb{Z})$ is a cyclic group of order 1 .

(ii) Now, suppose that $d \neq 1$. We denote by $\bar{x}$ the equivalence class of $x$ modulo $n \mathbb{Z}$ and $\dot{x}$ the equivalence class of $x$ modulo $m \mathbb{Z}$. Let $p \in \operatorname{hom}(\mathbb{Z} / n \mathbb{Z}, \mathbb{Z} / m \mathbb{Z})$ be a nonzero morphism. To define $p$, it suffices to know that $p(\overline{1})=\dot{k}$, where $k \in\{1,2, \ldots, m-1\}$. Since $n \overline{1}=\overline{0}$, we have $n \dot{k}=\dot{0}$. Thus $n k \equiv 0(\bmod m)$. Hence, by Remark 6.3, $k \equiv 0(\bmod (m / d))$, where $d=\operatorname{gcd}(m, n)$ so that there exists $t \in \mathbb{Z}$ such that $k=t(m / d)$. Let $\rho: \mathbb{Z} / n \mathbb{Z} \rightarrow \mathbb{Z} / m \mathbb{Z}$ be the morphism of groups defined by $\rho(\bar{x})=(m / d) \dot{x}$. Then $p=t \rho$. It follows that hom $(\mathbb{Z} /$ $n \mathbb{Z}, \mathbb{Z} / m \mathbb{Z})$ is a cyclic group generated by the morphism $\rho$. It is clear that $\rho$ is of or$\operatorname{der} d$.

Let us derive two important consequences of Proposition 6.4.

COROLLARY 6.5. The groups $\mathbb{Z} / n \mathbb{Z}$ and $\mathbb{Z} / m \mathbb{Z}$ are relatively terminal if and only if $\operatorname{gcd}(m, n)$ $=1$.

Corollary 6.6. Let $G, H$ be two finite Abelian groups. Then the following statements are equivalent:

(i) $G$ and $H$ are relatively terminal;

(ii) $\operatorname{gcd}(o(G), o(H))=1$.

Proof. It is well known that a finite Abelian group is in a unique manner a direct sum of primary cyclic groups (fundamental theorem of finite Abelian groups). Now, Remark 5.6 and Corollary 6.5 permit to check easily the equivalence $(\mathrm{i}) \Leftrightarrow$ (ii).

Problems 6.7. (1) Find two finite non Abelian groups $G, H$ which are relatively terminal and such that $\operatorname{gcd}(o(G), o(H)) \neq 1$.

(2) Let $G$ be a group not isomorphic to $\mathbb{Z}$. Is there a group $H(G)$ such that $G, H(G)$ are relatively terminal?

The following observation will be useful in the next theorem.

Remark 6.8. Let $(G,+)$ be a finite cyclic group generated by $\rho \in G$ and let $d$ be the order of $\rho$. Let $\alpha \in \mathbb{Z}$ and $p=\alpha \rho \in G$. Then, $p$ generates $G$ if and only if $\operatorname{gcd}(\alpha, d)=1$. 
We are now in a position to state the main result of this section.

Theorem 6.9. Let $(m, n) \in \mathbb{N}^{*} \times \mathbb{N}$ such that $\operatorname{gcd}(m, n) \neq 1$. Then the following statements are equivalent:

(i) $\mathbb{Z} / n \mathbb{Z}$ is $\mathbb{Z} / m \mathbb{Z}$-ifiable;

(ii) $m$ divides $n$.

Proof

Step 1. (ii) $\Rightarrow$ (i). We prove that the morphism $\mu: \mathbb{Z} / n \mathbb{Z} \rightarrow \mathbb{Z} / m \mathbb{Z}$ defined by $\mu(\bar{x})=\dot{x}$ is a $\mathbb{Z} / m \mathbb{Z}$-ification of $\mathbb{Z} / n \mathbb{Z}$. Let $f \in \operatorname{hom}(\mathbb{Z} / n \mathbb{Z}, \mathbb{Z} / m \mathbb{Z})$. Then there exists a unique $k \in$ $\{0,1, \ldots, m-1\}$ such that $f=k \mu$ (see the proof of Proposition 6.4). We denote by $\tilde{f}$ : $\mathbb{Z} / m \mathbb{Z} \rightarrow \mathbb{Z} / m \mathbb{Z}$ the morphism defined by $\tilde{f}(\dot{x})=k \dot{x}$. Then $\tilde{f} \circ \mu=f$. Now, let $g \in$ $\operatorname{hom}(\mathbb{Z} / n \mathbb{Z}, \mathbb{Z} / m \mathbb{Z})$ such that $g \circ \mu=f$. Then there exists a unique $a \in\{0,1, \ldots, m-1\}$ such that $g=a \mu$. Since $g \circ \mu=f$, we get $a x \equiv k x(\bmod m)$, for each $x \in \mathbb{Z}$. Hence $a \equiv k$ $(\bmod m)$ so that $a=k$, and then $g=\tilde{f}$.

Therefore, $\mu$ is a $\mathbb{Z} / m \mathbb{Z}$-ification of $\mathbb{Z} / n \mathbb{Z}$.

Step 2. Let $m \in \mathbb{N}^{*}$ and $n \in \mathbb{N}$ such that $\operatorname{gcd}(m, n) \neq 1$. If $p: \mathbb{Z} / n \mathbb{Z} \rightarrow \mathbb{Z} / m \mathbb{Z}$ is a $\mathbb{Z} / m \mathbb{Z}$ ification of $\mathbb{Z} / n \mathbb{Z}$, then $p$ generates the cyclic group hom $(\mathbb{Z} / n \mathbb{Z}, \mathbb{Z} / m \mathbb{Z})$.

Proof of Step 2. From the proof of Proposition 6.4, we know that hom $(\mathbb{Z} / n \mathbb{Z}, \mathbb{Z} / m \mathbb{Z})$ is the cyclic group generated by the morphism $\rho: \mathbb{Z} / n \mathbb{Z} \rightarrow \mathbb{Z} / m \mathbb{Z}$ defined by $\rho(\bar{x})=(m / d) \dot{x}$, with $d=\operatorname{gcd}(m, n)$. Thus, there exists $\alpha \in\{0,1, \ldots, d-1\}$ such that $p=\alpha \rho$. For each $f \in$ $\operatorname{hom}(\mathbb{Z} / n \mathbb{Z}, \mathbb{Z} / m \mathbb{Z})$, there exist a unique $\tilde{f} \in \operatorname{hom}(\mathbb{Z} / m \mathbb{Z}, \mathbb{Z} / m \mathbb{Z})$ such that $\tilde{f} \circ p=f$. On the other hand, there exists a unique $k \in\{0,1, \ldots, d-1\}$ and a unique $a_{k} \in\{0,1, \ldots, m-$

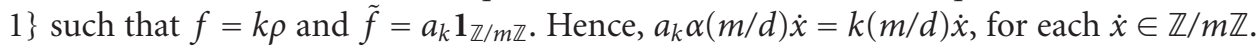
Thus $\alpha a_{k} \equiv k(\bmod d)$. In particular, for $f=\rho$, we get $k=1$, and then $\alpha a_{1} \equiv 1(\bmod d)$. By Remark 6.3, we have $\operatorname{gcd}(\alpha, d)=1$. Thus $p=\alpha \rho$ generates the cyclic group hom $(\mathbb{Z} / n \mathbb{Z}$, $\mathbb{Z} / m \mathbb{Z})$, by Remark 6.8 .

Step 3. (i) $\Rightarrow$ (ii). Let $p: \mathbb{Z} / n \mathbb{Z} \rightarrow \mathbb{Z} / m \mathbb{Z}$ be a $\mathbb{Z} / m \mathbb{Z}$-ification of $\mathbb{Z} / n \mathbb{Z}$. By Step 2 , $p$ generates the cyclic group hom $(\mathbb{Z} / n \mathbb{Z}, \mathbb{Z} / m \mathbb{Z})$. Thus, there exists $\alpha \in\{0,1, \ldots, d-1\}$ such $p=\alpha \rho$ and $\operatorname{gcd}(\alpha, d)=1$.

Now, let us prove that $m$ divides $n$. For $\beta \in\{0,1, \ldots, d-1\}$ and $f=\beta \rho$, there exists a unique $\tilde{f} \in \operatorname{hom}(\mathbb{Z} / m \mathbb{Z}, \mathbb{Z} / m \mathbb{Z})$ such that $\tilde{f} \circ p$. There exists $a_{\beta} \in\{0,1, \ldots, m-1\}$ such

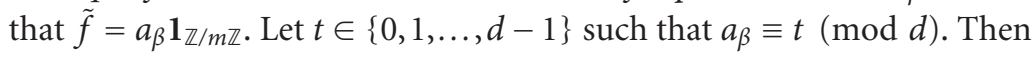

$$
t \mathbf{1}_{\mathbb{Z} / m \mathbb{Z}} \circ p=(t+d) \mathbf{1}_{\mathbb{Z} / m \mathbb{Z}} \circ p=f .
$$

Hence, by the uniqueness of morphism extending $f$ over $p$, we get $t \mathbf{1}_{\mathbb{Z} / m \mathbb{Z}}=(t+d) \mathbf{1}_{\mathbb{Z} / m \mathbb{Z}}$. This leads to the congruence $d \equiv 0(\bmod m)$. Therefore, $m$ divides $n$.

The following corollary gives examples of non-T-groups of arbitrary order and proves that the quotient of a $\mathbf{T}$-group need not be a $\mathbf{T}$-group.

Corollary 6.10. For each $n \in \mathbb{N}-\{0,1\}, \mathbb{Z} / n \mathbb{Z}$ is not a T-group in $\mathscr{H}$.

Proof. Let $p$ be a prime factor of $n$. By Theorem $6.9, \mathbb{Z} / p n \mathbb{Z}$ is $\mathbb{Z} / n \mathbb{Z}$-ifiable. If we suppose that $\mathbb{Z} / n \mathbb{Z}$ is a $\mathbf{T}$-group, then there exists a group $H$ such that $\mathbb{Z} / n \mathbb{Z}$ is $H$-terminal and $\mathbb{Z} / p n \mathbb{Z}$ is isomorphic to $\mathbb{Z} / n \mathbb{Z} \times H$. For a reason of cardinality, $H$ is a group of order $p$. 
Hence, $H$ is isomorphic to $\mathbb{Z} / p \mathbb{Z}$. It follows that $\mathbb{Z} / p n \mathbb{Z}$ is isomorphic to $\mathbb{Z} / n \mathbb{Z} \times \mathbb{Z} / p \mathbb{Z}$. Thus $\mathbb{Z} / n \mathbb{Z} \times \mathbb{Z} / p \mathbb{Z}$ is a cyclic group. But it is a part of the folklore of algebra that the direct product of two cyclic groups is cyclic if and only if their order are relatively prime. Therefore, $\mathbb{Z} / n \mathbb{Z}$ is not a $\mathbf{T}$-group in $\mathcal{H}$.

\section{Acknowledgment}

The authors are grateful to the referees for constructive comments and suggestions on the first version of this paper.

\section{References}

[1] E. Bouacida, O. Echi, G. Picavet, and E. Salhi, An extension theorem for sober spaces and the Goldman topology, Int. J. Math. Math. Sci. 2003 (2003), no. 51, 3217-3239.

[2] E. Bouacida, O. Echi, and E. Salhi, Foliations, spectral topology, and special morphisms, Advances in Commutative Ring Theory (Fez, 1997), Lecture Notes in Pure and Appl. Math., vol. 205, Marcel Dekker, New York, 1999, pp. 111-132.

[3] - Feuilletages et topologie spectrale [Foliations and spectral topology], J. Math. Soc. Japan 52 (2000), no. 2, 447-464.

[4] C. Casacuberta and A. Frei, On saturated classes of morphisms, Theory Appl. Categ. 7 (2000), no. $4,43-46$.

[5] C. Casacuberta, A. Frei, and G. C. Tan, Extending localization functors, J. Pure Appl. Algebra 103 (1995), no. 2, 149-165.

[6] C. Cassidy, M. Hébert, and G. M. Kelly, Reflective subcategories, localizations and factorization systems, J. Austral. Math. Soc. Ser. A 38 (1985), no. 3, 287-329.

[7] Y. S. Cho, On reflective subcategories, Kyungpook Math. J. 18 (1978), no. 2, 143-146.

[8] J. Činčura, Closed structures on reflective subcategories of the category of topological spaces, Topology Appl. 37 (1990), no. 3, 237-247.

[9] A. Deleanu, A. Frei, and P. Hilton, Generalized Adams completion, Cahiers Topologie Géom. Différentielle 15 (1974), 61-82.

[10] S. E. Dickson, A torsion theory for Abelian categories, Trans. Amer. Math. Soc. 121 (1966), 223235.

[11] O. Echi, A topological characterization of the Goldman prime spectrum of a commutative ring, Comm. Algebra 28 (2000), no. 5, 2329-2337.

[12] Q Quasi-homeomorphisms, Goldspectral spaces and Jacspectral spaces, Boll. Unione Mat. Ital. Sez. B Artic. Ric. Mat. (8) 6 (2003), no. 2, 489-507.

[13] R. El Bashir and J. Velebil, Simultaneously reflective and coreflective subcategories of presheaves, Theory Appl. Categ. 10 (2002), no. 16, 410-423.

[14] A. Frei, On completion and shape, Bol. Soc. Brasil. Mat. 5 (1974), no. 2, 147-159.

[15] P. J. Freyd and G. M. Kelly, Categories of continuous functors. I, J. Pure Appl. Algebra 2 (1972), no. 3, 169-191.

[16] A. Grothendieck and J. A. Dieudonné, Eléments de géométrie algébrique, Springer, New York, 1971.

[17] J. M. Harvey, Reflective subcategories, Illinois J. Math. 29 (1985), no. 3, 365-369.

[18] H. Herrlich, Topologische Reflexionen und Coreflexionen, Lecture Notes in Mathematics, no. 78, Springer, Berlin, 1968.

[19] - Almost reflective subcategories of Top, Topology Appl. 49 (1993), no. 3, 251-264.

[20] H. Herrlich and G. E. Strecker, H-closed spaces and reflective subcategories, Math. Ann. 177 (1968), 302-309. 
3404 The envelope of a subcategory in topology and group theory

[21] M. Hochster, Prime ideal structure in commutative rings, Trans. Amer. Math. Soc. 142 (1969), 43-60.

[22] D. Holgate, Linking the closure and orthogonality properties of perfect morphisms in a category, Comment. Math. Univ. Carolin. 39 (1998), no. 3, 587-607.

[23] P. T. Johnstone, Stone Spaces, Cambridge Studies in Advanced Mathematics, vol. 3, Cambridge University Press, Cambridge, 1982.

[24] G. M. Kelly, On the ordered set of reflective subcategories, Bull. Austral. Math. Soc. 36 (1987), no. 1, 137-152.

[25] S. MacLane, Categories for the Working Mathematician, Graduate Texts in Mathematics, vol. 5, Springer, New York, 1971.

[26] K. Morita, Čech cohomology and covering dimension for topological spaces, Fund. Math. 87 (1975), 31-52.

[27] _ Dimension of general topological spaces, Surveys in General Topology (G. M. Reed, ed.), Academic Press, New York, 1980, pp. 297-336.

[28] J. R. Porter, Extension function and subcategories of HAUS, Canad. Math. Bull. 18 (1975), no. 4, 587-590.

[29] J. Rada, M. Saorín, and A. del Valle, Reflective subcategories, Glasg. Math. J. 42 (2000), no. 1, 97-113.

[30] L. Stramaccia, Reflective subcategories and dense subcategories, Rend. Sem. Mat. Univ. Padova 67 (1982), 191-198.

[31] W. Tholen, Reflective subcategories, Topology Appl. 27 (1987), no. 2, 201-212.

Ahmed Ayache: Department of Mathematics, College of Sciences, University of Bahrain, P.O. Box 32038, Isa Town, Kingdom of Bahrain

E-mail address: aayache@sci.uob.bh

Othman Echi: Department of Mathematics, Faculty of Sciences, Tunis-El Manar University, Campus Universitaire, 2092 Tunis, Tunisia

E-mail addresses: othechi@yhoo.com; othechi@math.com 


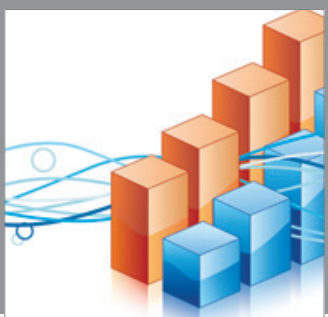

Advances in

Operations Research

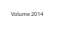

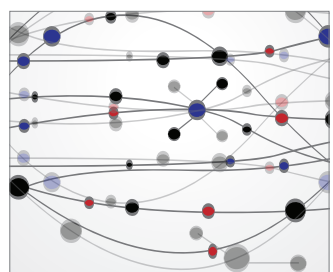

\section{The Scientific} World Journal
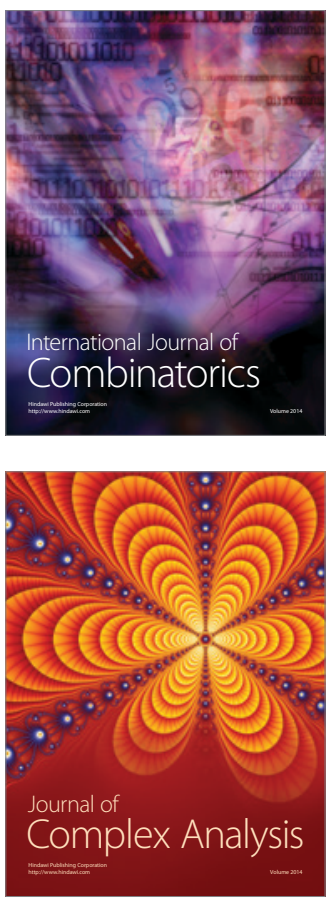

International Journal of

Mathematics and

Mathematical

Sciences
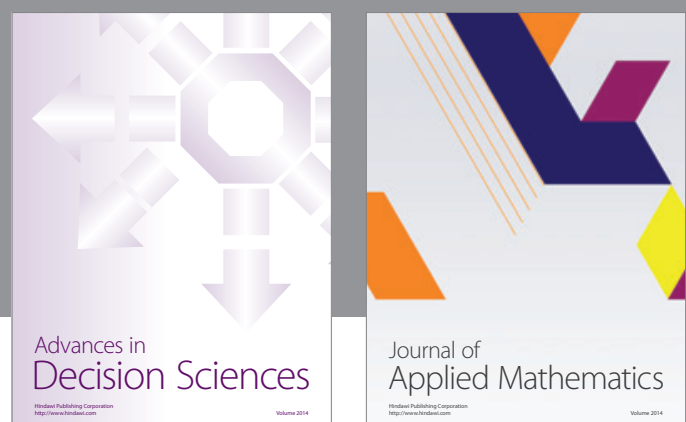

Journal of

Applied Mathematics
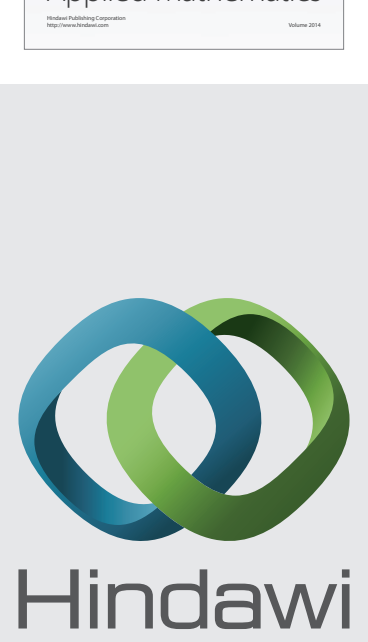

Submit your manuscripts at http://www.hindawi.com
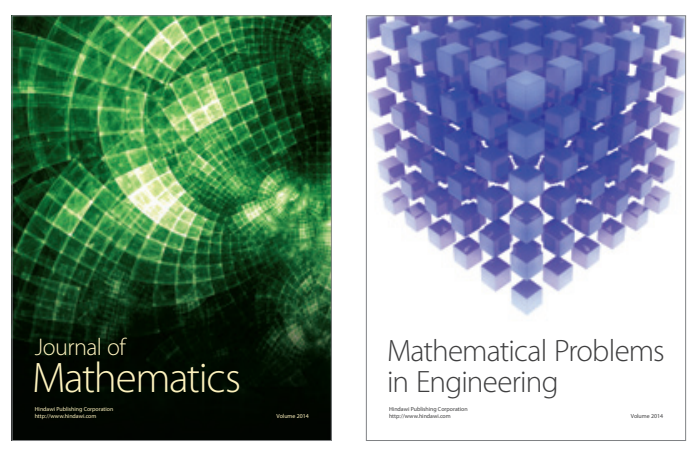

Mathematical Problems in Engineering
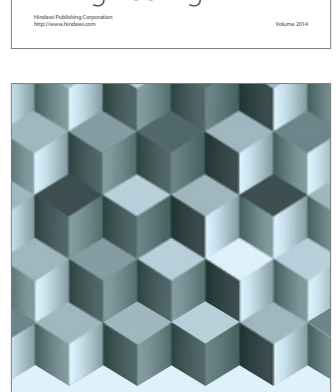

Journal of

Function Spaces
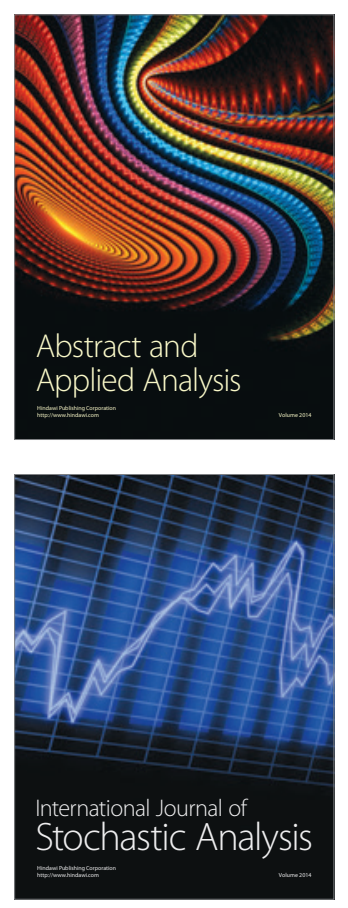

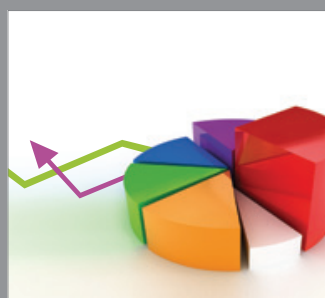

ournal of

Probability and Statistics

Promensencen
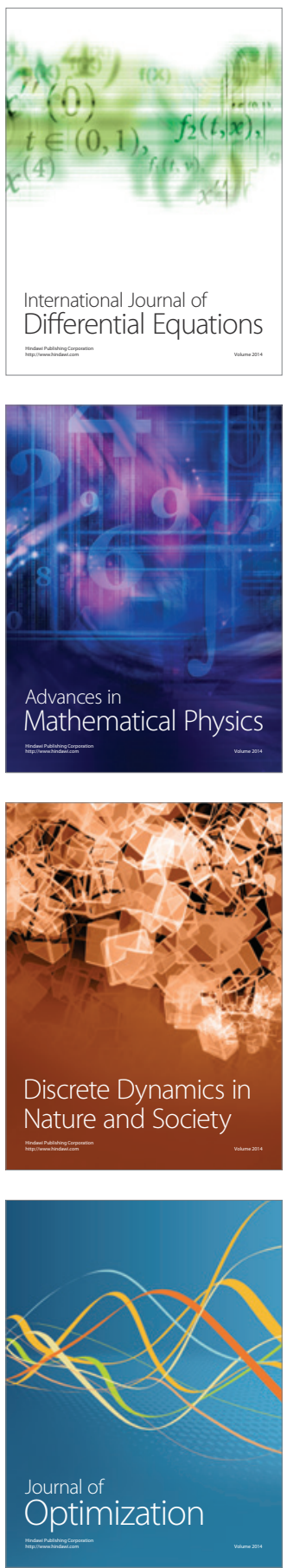